\title{
Guangxi 's Logistics Distribution Network Based on the Theory of the Uncertainty Decision-making Model
}

\author{
Qing HUA ${ }^{1, a,{ }^{*}}$, Zhi-Gao LIAO ${ }^{1, b}$, Sheng-Jie TANG ${ }^{1, c}$, Hui QIU ${ }^{1, d}$ \\ ${ }^{1}$ Guangxi University Of Science and Technology China department of \\ management LiuZhou 545006 \\ ${ }^{*}$ Corresponding author \\ a1406748247@qq.com, ${ }^{\mathrm{b}}$ liaozhigao126.com, ${ }^{\mathrm{c} 913005783 @ q q . c o m,{ }^{d} 358948635 @ q q . c o m}$
}

Keywords: Guangxi's Intercity Logistics, Demand Uncertainty, Vehicle Routing Optimization, Saving Algorithm

\begin{abstract}
In the area of modern physical distribution, vehicle routing problem has become the focal point in the research. During the process of transportation, efficient and the reasonable vehicles travel route not only can reduce the overall cost significantly, but also improve customer's satisfaction. This paper base on the current situation of Guangxi inter-city logistics and the conditions of demand uncertainty. It takes advantage of the saving algorithm to solve modeling problem, which will greatly improve the efficiency of logistics distribution.
\end{abstract}

\section{Introduction}

There are many optimization decision problems in logistics and distribution business, among them, the vehicle routing problem is the focus of research. Research on this field, Tillman first proposed algorithm to solve the vehicle routing problem with stochastic demand. Powell and others focused on research that based on the uncertainty of the planning model. Yao-Huang Guo and other people have studied two pre-optimized strategies which under the circumstances of customer demand inalienably in view of demand indefinite VFR. Besides, they have revised the genetic algorithm and the simulation annealing algorithm which was used in solving. In previous researches, researchers seldom took uncertainties into consideration, which will lead to deviating with reality to a certain degree. Based on previous studies, we introduced the demand uncertainty factor to create a vehicle route planning model in the environment and solved the problem.

\section{Model Description}

In numerous vehicle route planning studies, each node demand is known. The distribution plan is made in a static environment. However, it will be affected by a lot of constraints in practice. In view of above situation, we made a research on the basis of the traditional route planning and take into account of that the city demand obeys some distribution and satisfaction of a service levels.

\section{Model Assumptions}

Firstly, There is only road transportation as the mode of transport in the enclosed distribution center.

Secondly, all vehicles regard the distribution center as their starting point and end point.

Thirdly, considering only one vehicle model, which with the same carrying capacity, the same volume restrictions, and the same mileage.

Fourthly, take no account of "Empty Phenomenon".

Fifthly, the city has safety stock. The demand of today at least is know at previous day, and the stock of the day before should be greater than the demand for the next day, what's more, it's to be known to the initial inventory of the clients. 


\section{Model Buildup}

A distribution center needs to distribute a same kind of goods to $\mathrm{N}$ cities , the distance between the cities and the distance of cities to the distribution center are known, each city needs to obey a known distribution $\mathrm{F}$, the distribution center hope that the service rate of cities distribution remained above the level of q. Making a distribution arrangement for the distribution center, which can make distribution center arrange fluently according to the distribution plan as long as the city demands comply with the known distribution.

\section{Related Symbols and Parameters:}

$\mathrm{K}$ : a collection of available vehicles. $\mathrm{k}$ belongs to $\mathrm{M}$

Q: maximum load truck

Hij: Vehicles from city $\mathrm{i}$ to city $\mathrm{j}$ transportation mileage. $\mathrm{i}, \mathrm{j}$ belongs to $\mathrm{V}, \mathrm{i}$ is not equal to $\mathrm{j}$

C0: a car fixed costs out of the bus

C1: unit transportation cost of transportation mileage

fi: demanding of city i are submitted to distribution density function

$\mu$ : the city i's requirements are obeyed to mean distribution functions

$\sigma i$ : the city i demand to obey the standard deviation of the distribution function

q: the service level of the city'

$\mathrm{V}: \mathrm{V}=(1,2,3 \ldots \mathrm{n})$ represents the collection of Sites

\section{Decision Variables:}

Pik: vehicle $\mathrm{k}$ delivers a amount of goods for the city $\mathrm{i}$

$x_{\mathrm{ijk}}=1$ vehicle $\mathrm{k}$ delivers the goods for the city $\mathrm{i}$. $\mathrm{k}$ belongs to $\mathrm{M}, \mathrm{i}$ belong to $\mathrm{V}$

$0 \mathrm{k}$ is not deliver for the city $\mathrm{i}$. $\mathrm{k}$ belongs to $\mathrm{M}$, i belong to $\mathrm{V}$

\section{Mathematical Model}

\section{The Objective Function}

$\operatorname{Min}\left(\sum_{i=0}^{n} \sum_{k=1}^{M} \sum_{\substack{i=1 \\ j=0}}^{n} x_{\mathrm{ij} k} \cdot C_{o}+\sum_{k=1}^{M} \sum_{i=o}^{n} \sum_{\substack{j=0 \\ i=1}}^{n} x_{i j k} \cdot H_{i j} \cdot C_{1}\right)$

\section{Constraints:}

$\sum_{i=1}^{n} P_{i k} \leq Q, \forall k$

$\sum_{1=0}^{n} x_{i j k}=\sum_{1=0}^{n} x_{j i k}, \forall j, k$

$\sum_{i=0}^{n} \sum_{k=1}^{M} x_{i j k} \leq 1, \forall j$ 


$$
\begin{aligned}
& X_{i j k} \in\{0,1\}, \forall i, j, k, i \neq j \\
& \sum_{i=1}^{n} P_{i k} \geq \sum_{j=0}^{n} \sum_{k=1}^{M} \sum_{i=1}^{n} u_{i} * x_{i j k}+z * \sqrt{\sum_{i=1}^{n} \sigma * x_{i j k}, \forall k}
\end{aligned}
$$

Among it, Eq. 3explainsthe flow balance constraint: Eq.4 is to require each city has no more than a car for service: Eq. 6 is a city service rate requirement constraint that the total load of the vehicle $\mathrm{k}$ must be greater than it is responsible distribution services in all cities in the q-level minimum requirements, in which $\mathrm{Z}$ is the level of the distribution corresponding to the service position on the points of $\mathrm{q}$.

\section{Case Study}

To build a logistics distribution center in NanNing of GuangXi by Assumptions, distribute to thirteen cities of GuangXi, the demand of the city branch both obey the normal distribution, and the demand between the city outlets is independent of each other. In order to ensure the demand, logistics distribution center for network service level is no less than ninety-five percent, the maximum load for vehicles is one hundred,we need to choose the logistics distribution center distribution scheme now.

Table 1 The demand of thirteen cities of Guang Xi

\begin{tabular}{|l|l|l|l|l|l|l|l|}
\hline city & 1 & 2 & 3 & 4 & 5 & 6 & 7 \\
\hline$\left(\mu, \sigma^{2}\right)$ & $(18,6)$ & $(21,4)$ & $(19,8)$ & $(23,5)$ & $(20,7)$ & $(17,8)$ & $(25,7)$ \\
\hline city & 8 & 9 & 10 & 11 & 12 & 13 & \\
\hline$\left(\mu, \sigma^{2}\right)$ & $(22,8)$ & $(27,9)$ & $(24,4)$ & $(22,9)$ & $(21,5)$ & $(23,3)$ & \\
\hline
\end{tabular}

Table 2 Thirteen cities of GuangXi and logistics distribution center distance ( 1 for distribution center)

\begin{tabular}{|l|l|l|l|l|l|l|l|l|l|l|l|l|l|}
\hline & 1 & 2 & 3 & 4 & 5 & 6 & 7 & 8 & 9 & 10 & 11 & 12 & 13 \\
\hline 1 & 0 & & & & & & & & & & & & \\
\hline 2 & 174 & 0 & & & & & & & & & & & \\
\hline 3 & 460 & 313 & 0 & & & & & & & & & & \\
\hline 4 & 469 & 322 & 39 & 0 & & & & & & & & & \\
\hline 5 & 437 & 290 & 47 & 34 & 0 & & & & & & & & \\
\hline 6 & 433 & 312 & 102 & 178 & 147 & 0 & & & & & & & \\
\hline 7 & 442 & 323 & 243 & 230 & 197 & 66 & 0 & & & & & & \\
\hline 8 & 205 & 96 & 305 & 300 & 268 & 228 & 237 & 0 & & & & & \\
\hline 9 & 237 & 196 & 379 & 366 & 331 & 260 & 269 & 118 & 0 & & & & \\
\hline 10 & 67 & 140 & 444 & 453 & 430 & 416 & 425 & 181 & 170 & 0 & & & \\
\hline 11 & 309 & 353 & 581 & 568 & 538 & 464 & 473 & 322 & 204 & 242 & 0 & & \\
\hline 12 & 318 & 436 & 740 & 743 & 711 & 637 & 646 & 481 & 377 & 297 & 202 & 0 & \\
\hline 13 & 110 & 257 & 570 & 579 & 547 & 543 & 552 & 315 & 347 & 177 & 380 & 314 & 0 \\
\hline
\end{tabular}

Table 3 Vehicle related cost data

\begin{tabular}{|l|l|}
\hline The unit cost of transportation & The unit fixed costs of the truck \\
\hline five RMB/kilometers & six hundred RMB/truck \\
\hline
\end{tabular}

Assuming that logistics distribution center is not optimized route arrangement,so we need to use the vehicle twelve times to complete all of the city branch distribution task at a time,the total mileage 
is 7322 kilometers, the total distribution costs is $36610 \mathrm{RMB}$, the fare is $7800 \mathrm{RMB}$ and the operating cost is $44410 \mathrm{RMB}$.

\section{Solution of the model results}

According to the theory of probability, $X \sim N\left(\mu, \sigma^{2}\right)$, When the service level is ninety-five percents $X=\mu+Z * \sigma$, Check the normal distribution table $Z=Z_{0.5}=1.65$.Using the saving algorithm to solve the model, take the corresponding data generation into the model calculation .

Table 4 The demand of each city by ninety-five percent of service level

\begin{tabular}{|l|l|l|l|l|l|l|}
\hline 1 & 2 & 3 & 4 & 5 & 6 & 7 \\
\hline 22.0 & 24.3 & 23.7 & 26.7 & 24.4 & 21.7 & 29.4 \\
\hline 8 & 9 & 10 & 11 & 12 & 13 & \\
\hline 26.7 & 32.0 & 27.3 & 27.0 & 24.7 & 25.9 & \\
\hline
\end{tabular}

We plan the customer to the different transport routes, provided the delivery service by the same truck, the purpose of the merger customer is to save the maximum distance. This is a repeat procedure.

We follow two principles here: First, the merger of two routes is feasible, and the distribution amount is not greater than the maximum load of the truck .Second, try to take the largest saving amount of the merged into a new feasible line. This process continued until the new merger is over.

First of all, the biggest save distance from cities three and four of 890, and this kind of merger is feasible. The total traffic volume is $23.7+26.7=50.4$, and the truck load two hundred, so it classified two customers as a line, We don't need to consider the save distance 890 in next step.

Repeated the process, no longer considered the merged line, and the rest is merged in turn. The last line merger of the city network is classified as two roads, showing in the following Table 5.

Table 5 Calculate the delivery route arrangements

\begin{tabular}{|l|l|l|l|l|}
\hline distribution route & $1-4-3-5-2-1$ & $1-6-7-9-1$ & $1-12-11-8-1$ & $1-10-1$ \\
\hline distribution amount & 99.1 & 83.1 & 78.4 & 27.3 \\
\hline
\end{tabular}

From the Table 5, We need four trucks to complete all distribution of the city branch. According to the related cost data of vehicle and the distance between each city branch, From the distribution line arrangement, the total mileage is 2987 kilometers, the total cost is $17335 \mathrm{RMB}$, the operation cost is $14935 \mathrm{RMB}$, the fare of the vehicle is 2400RMB.Compared with the initial distribution network distribution ,new distribution route save 4335 kilometers, total cost save 19275 RMB.

\section{Acknowledgement}

This research was financially supported by the College Students ' innovation and Entrepreneurship Program (2012-42).

\section{References}

[1]Liu Ping, Zhou D H. Study on Robust Fault Toleran Control of Uncertain Linear Time-delay Systems[J]Control Theory and Application, 2003, 20( 1):78-80.

[2] Xian-Jun Zhang, Uncertain Transportation Problem Model and Algorithm [D]. Chongqing University, 2006.

[3] Wen-Jia Chen, Saving mileage method in the production enterprise logistics application [J]. Commercial culture (second half). 2011 (11). 
[4] Ying-Ming Zhang ,Based conservation mileage France logistics distribution route optimization and improvement of research [J]. Logistics Technology. 2011 (04).

[5] Lee as Jiao. "Saving mileage Law" in a logistics company distribution center in the practical application [J]. Scientific and technological information. 2008 (28).

[6] Yao-Huang Guo, Bing-Lei Xie, Guo Qiang.Dynamic vehicles route question: Present situation and forecast. [J] systems engineering theory method application, 2002,11(2):116-120.

[7] Yao-Huang Guo, Uncertain information under dynamic vehicle routing. [J] academic trends, 2004,2:24.

[8] Yao-Huang Guo, Xiao-Peng Zhong, Dynamic vehicles route question line model analysis, [J] management science journal, 2006,9(1):33-35.

[9] Yao-Huang Guo, Bing-Lei Xie. A class of stochastic dynamic vehicle routing problem with strategic analysis. Engineering and Engineering Management. 2003, 4:114-115.

[10] Jian-Yong Zhang, Li Jun, Yao-Huang Guo. Real-time dynamic vehicle scheduling problem under fuzzy demand information problems. Journal of management engineering. 2004, 4:69-72. 